\title{
Inhibition of liver fibrosis using vitamin A-coupled liposomes to deliver matrix metalloproteinase-2 siRNA in vitro
}

\author{
YIPING LI ${ }^{1 *}$, FENG LIU $^{1 *}$, FENGAN DING $^{1}$, PINGSHENG CHEN $^{1}$ and MENG TANG ${ }^{2}$ \\ ${ }^{1}$ Department of Pathology, Medical School of Southeast University; ${ }^{2}$ Department of Toxicology, \\ School of Public Health, Southeast University, Nanjing, Jiangsu 210009, P.R. China
}

Received August 11, 2014; Accepted April 24, 2015

DOI: $10.3892 / \mathrm{mmr} .2015 .3842$

\begin{abstract}
Hepatic fibrosis is a common form of wound healing in response to chronic liver injuries and can lead to more serious complications, including mortality. It is well-established that hepatic stellate cells (HSCs) are central mediators of hepatic fibrosis, and matrix metalloproteinase-2 (MMP-2) is important in the formation of liver fibrosis. In addition, HSCs are the primary cells secreting MMP-2 and extracellular matrix, therefore, there has been increasing interest in developing agents with high selectivity towards HSCs. However, no clinical drugs based on MMP-2, directed against HSCs, have been used to prevent fibrosis. Following consideration of the abundant vitamin A (VitA) receptors expressed on the cellular membrane of HSCs, the present study constructed VitA-coupled liposomes (VitA-lips) using dicyclohexylcarbodiimide-1, 3-diaminopentane condensation, rotatory film processing and ultrasonic oscillation. The results revealed that the liposomes exhibited low cytotoxicity and a suitable binding ability to MMP-2 small interference (si)RNA. Furthermore, the liposomes effectively delivered MMP-2 siRNA to the HSC-T6 cells. When HSCs were treated with the liposomes carrying MMP-2 siRNA (VitA-lip-MMP-2 siRNA), the mRNA expression and activity of MMP-2, and the protein expression levels of $\alpha$-smooth muscle actin and type I collagen were significantly reduced. These results suggested that inhibition of the expression of MMP-2 in HSC-T6 cells may contribute to preventing hepatic fibrosis, and provided experimental support to the development of specific drugs against MMP-2 to prevent fibrogenesis in chronic liver disease.
\end{abstract}

Correspondence to: Professor Pingsheng Chen, Department of Pathology, Medical School of Southeast University, Dingjiaqiao 87, Nanjing, Jiangsu 210009, P.R. China

E-mail: chenps@seu.edu.cn

${ }^{*}$ Contributed equally

Key words: cationic liposomes, hepatic stellate cells, liver fibrosis, matrix metalloproteinase-2, small interference RNA

\section{Introduction}

Hepatic fibrosis, a prominent pathological feature at the end stage of chronic liver disease, is a dynamic process and develops due to an increase in extracellular matrix (ECM) synthesis and deposition, along with insufficient remodeling, however, fibrosis may be reversible prior to the establishment of advanced architecture (1-3). Matrix metalloproteinase-2 (MMP-2) may be involved in the formation and reversal of hepatic fibrosis (4-6). MMP-2 promotes the development of fibrosis in the early stage, but increased MMP-2 activity can induce the reversal of fibrosis following removal of the pathogenic factors $(4,6)$. However, whether MMP-2 has an effect on the formation of hepatic fibrosis by directly examining hepatic stellate cells (HSCs) remains to be elucidated, and no clinical antifibrotic drugs based on MMP-2 have been approved for preventing fibrosis $(5,7)$.

RNA interference (RNAi) is a strategy, which involves specifically degrading homogenous mRNA to suppress its gene expression. Compared with other techniques for inhibiting gene expression, this method is not only specific, effective and persistent, but is also simple and safe. However, the vectors for siRNA have certain bottlenecks, including low transfer efficiency and inevitable prominent toxicity. Among these vectors, cationic liposomes are notable due to their lack of immunogenicity and natural degradation, however, specific targeting remains a challenge (8).

Several studies have demonstrated that hepatic stellate cells (HSCs) are crucial mediators of fibrosis $(3,9,10)$. HSCs reside in the perisinusoidal space and store vitamin A (VitA) in their quiescent state (11). The present study selected HSCs as target cells of fibrosis to analyze the effect of VitA-coupled liposomes (VitA-lips) combined with MMP-2 siRNA, which may aid further investigations examining specific fibrosis prophylactic drug treatments.

\section{Materials and methods}

Materials. Cholesterol (Aobox, Peiking, China), 3 $\beta-[\mathrm{N}$ -(N',N'-dimethylaminoethane) carbamoyl] cholesterol (DC-Chol; Sigma-Aldrich, St. Louis, MO, USA), 1,2-Dioleoyl-sn-glycero-3-phosphoethanolamine (DOPE; Sigma-Aldrich), MMP-2-siRNA (GenePharma, Shanghai, China), 4',6-diamidino-2-phenylindole (DAPI) staining 
solution (Beyotime Institute of Biotechnology, Haimen, China), mouse anti-human $\alpha$-SMA monoclonal antibody (cat. no. ZM-0003), rabbit anti-mouse type I collagen monoclonal antibody (cat. no. ZA-0616) and immunohistological reagent kits (Zhongshan Golden Bridge Biotechnology Co., Ltd., Beijing, China), gelatin zymography assay kits (Applygen Technologies, Inc., Beijing, China), H2600 transmission electronic microscope (TEM; JEM-200CX; JEOL, Ltd., Tokyo, Japan), Zeta Sizer 3000 laser particle size analyzer (Malvern Instruments Ltd., Malvern, UK), high-pressure homogenizer (Avestin, Ottawa, Canada), and fluorescent microscope (TI-S; Nikon Corporation, Tokyo, Japan) and an inverted fluorescent microscope (TE2000-U, Nikon Corporation). were all used. The hepatic stellate cell line HSC-T6 was purchased from the Cell Bank of the Chinese Academy of Sciences (Shanghai, China).

Cell culture. The HSC-T6 rat hepatic stellate cells were grown in Dulbecco's modified Eagle's medium (DMEM, Gibco Life Technologies, Carlsbad, CA, USA), supplemented with $10 \%$ (v/v) fetal bovine serum (Gibco Life Technologies) and antibiotics $(100 \mathrm{U} / \mathrm{ml}$ streptomycin and $100 \mathrm{U} / \mathrm{ml}$ penicillin) in plastic culture flasks or dishes at $37^{\circ} \mathrm{C}$ in a humidified incubator under a $5 \% \mathrm{CO}_{2}$ atmosphere, and the culture medium was replaced with fresh medium every other day. The confluent cells were then harvested with $0.25 \%$ trypsin-EDTA solution.

Preparation of cationic liposomes. DC-Chol, DOPE and cholesterol (4:3:3, molar ratio) were mixed in round-bottom flasks, and $\mathrm{CCl}_{3}-\mathrm{CH}_{3} \mathrm{OH}(3: 2, \mathrm{v} / \mathrm{v})$ was added to the mixture, followed by sonication for $5 \mathrm{~min}$ in a water bath prior to evaporation in $37^{\circ} \mathrm{C}$ to remove organic reagents. Finally, homogenous thin films were obtained from the internal walls of the flasks. The films were resolved with $20 \mathrm{ml}$ phosphate-buffered saline (PBS; pH 7.2), followed by centrifugation for $30 \mathrm{~min}$ at room temperature (RT). When the films were completely stripped down, they were sonicated for $1 \mathrm{~min}$ and then squeezed three times through $220 \mathrm{~nm}$ filters (Merck Millipore, Darmstadt, Germany) to reduce their size. Simultaneously, VitA-cephalin was synthesized using the dicyclohexylcarbodiimide-1, 3-diaminopentane (DCC-DAMP) method. Briefly, $50 \mathrm{mg}$ VitA (Zhengzhou Lion Biological Technology Co., Ltd., Henan, China) and $100 \mathrm{mg}$ phycoerythrin (Sigma-Aldrich) were dissolved in $5 \mathrm{ml}$ dimethyl sulfoxide (DMSO; Sigma-Aldrich), and mixed with $100 \mu$ l DAMP (Sinopharm Chemical Reagent Beijing Co., Ltd., Beijing, China), followed by the activation at $4^{\circ} \mathrm{C}$ for $30 \mathrm{~min}$. Subsequently, DCC (Sinopharm Chemical Reagent Beijing Co., Ltd.) solution (50 mg in $1 \mathrm{ml}$ chloroform) was slowly added to the above mixture, and agitated for $24 \mathrm{~h}$ at RT in the dark. After standing for $2 \mathrm{~h}$ at RT, the supernatant were mixed with $20 \mathrm{ml}$ cold acetone (Sinopharm Chemical Reagent Beijing Co., Ltd.), and centrifuged for $10 \mathrm{~min}$ at $9,279 \mathrm{x} g$ at RT. The precipitate was washed with cold acetone for three times and dried at RT, then the products were analyzed using a Fourier Transform Infrared spectrometer (Avatar 370 Thermo Nicolet; Thermo-Nicolet Corporation, Madison, WI, USA).

Characterization of cationic liposomes. Cationic liposomes were diluted and dropped onto copper gauze with a membrane for morphological characterization. The particle size was measured using a Zeta Sizer 3000 laser particle size analyzer and processed using Dynamic Light Scattering software (Malvern Instruments Ltd.). The average particle size and polydispersity index (PDI) were recorded. No aggregation or deposition were observed in the cationic liposomes on being maintained at RT for 2 months, and the stability was further examined using TEM. Briefly, a few drops of the liposome solution were dropped onto a TEM grid, dried and absorbed the extra solution with filter paper prior to analysis. TEM images were photographed using a field emission JEM-200CX TEM equipped with a charge-coupled device camera.

Reverse transcription-quantitative polymerase chain reaction (RT-qPCR). The VitA-lip-MMP-2 siRNA complex (mass ratio of cationic liposome to MMP-2-siRNA, 5:1; siRNA, $30 \mathrm{nM}$ ) was used to transfect the HSC-T6 cells at a density of $70-80 \%$. Subsequent to the cells being transfected for $36 \mathrm{~h}$ at $37^{\circ} \mathrm{C}$, the total RNA was extracted using TRIzol ${ }^{\circledR}$ reagent (Invitrogen Life Technologies, Carlsbad, CA, USA), and the mRNA expression of MMP-2 was analyzed using RT-qPCR. Briefly, $1 \mu \mathrm{g}$ total RNA was reverse transcribed in a reaction mix containing $2 \mathrm{ml}$ dithiothreitol $(0.1 \mathrm{M}), 1 \mu \mathrm{l}$ dNTPs $(100 \mathrm{mM}), 2 \mu \mathrm{g}$ random hexamers, $1 \mu \mathrm{l}$ (200 units) superscript II reverse transcriptase and $1 \mu \mathrm{l}$ (40 units) RNAse inhibitor (GE Healthcare, Chalfont, UK) for $1 \mathrm{~h}$ at RT, then the synthesized cDNA was used for RT-qPCR. The sequences of the sense and antisense primers for MMP-2 were 5'-CATCGTACTCCTCGT TGCTGATCCACAT-3' and 5'-CTCCCTCATGCCATCCTG CGTCTG-3', respectively. To normalize the loading samples, a GAPDH control was produced using sense 5'-GAAGGGCTC ATGACCACAGT-3' and antisense 5'-GGATGCAGGGAT GATGTTCT-3' primers. The primers were synthesized by Yingjun Biotechnogy Co., Ltd. (Shanghai, China). The PCR amplification cycles were as follows: $94^{\circ} \mathrm{C} 5 \mathrm{~min}$, followed by 35 cycles of $94^{\circ} \mathrm{C}$ for $30 \mathrm{sec}, 62.5^{\circ} \mathrm{C}$ for $30 \mathrm{sec}$ and $72^{\circ} \mathrm{C}$ for $30 \mathrm{sec}$, with a final extension step for $10 \mathrm{~min}$ at $72^{\circ} \mathrm{C}$. The product was detected using electrophoresis and a $2 \%$ agarose gel (Sigma-Aldrich). The bands were scanned to analyze the gray values using a Smart Biological Electrophoretic Image Analyzer (Shanghai Furi Science \& Technology Co. Ltd., Shanghai, China). The expression of MMP-2 was normalized to the corresponding GAPDH band (117 bp). The interference efficiency of MMP-2 was calculated according to the following formula: Interference efficiency $=(1-$ MMP-2 expression in the siRNA group/MMP-2 expression in the untreated group) $\mathrm{x} 100 \%$.

Analysis of siRNA-binding ability. The cationic liposomes were mixed with siRNA $(0.01 \mathrm{~g} / \mathrm{l})$ at various mass ratios of cationic liposome to siRNA $(0: 1,1: 1,3: 1,5: 1,7: 1,9: 1$ and 15:1) and incubated at RT for $30 \mathrm{~min}$ to allow for complete electrostatic interaction between the liposomes and siRNA. Subsequently, the complexes were obtained and loaded into an agarose gel to evaluate the siRNA-binding ability of the cationic liposomes. The bands were scanned to analyze the gray values using a Smart Biological Electrophoretic Image Analyzer. The binding abilities at each mass ratio were calculated according to the following formula: (Gray value at $0: 1$ - gray value at other mass ratio)/(gray value of $0: 1) \times 100 \%$. 
Cell viability assay. An MTT assay was used to examine the cellular toxicity of the synthesized liposomes on HSC-T6 cells. The cells in the logarithmic growth phase were seeded on a 96 -well plate $\left(5 \times 10^{3} /\right.$ well $)$ and cultured for $24 \mathrm{~h}$. Experimental wells were used for cationic liposome/siRNA complexes with different mass ratios, whereas wells containing fresh DMEM media and Lipofectamine 2000 were used as negative and positive controls, respectively. For each group, six parallel wells were prepared. Following incubation for $72 \mathrm{~h}$ at $37^{\circ} \mathrm{C}$, MTT solution $20 \mu \mathrm{l}(5 \mathrm{~g} / \mathrm{l})$ was added to each well and after $4 \mathrm{~h}$, the culture reaction was terminated using $150 \mu \mathrm{l}$ DMSO. The optical density values were read at $492 \mathrm{~nm}$ using a microplate reader (Thermo Multiskan MK3; Thermo Fisher, Vantaa, Finland), with the viability of the cells presented as the percentage compared with the negative control cells.

Evaluation of transfection efficiency. The HSC-T6 cells were plated onto a 6 -well plate (3x10 $/$ well). At a density of $\sim 50-60 \%$, $0.2 \mathrm{ml}$ cationic liposome/carboxyfluorescein (FAM)-siRNA complex with different mass ratios $(0: 1,1: 1,3: 1,5: 1,7: 1,9: 1$, and $15: 1$, the concentration of siRNA was always $0.01 \mathrm{mg} / \mathrm{ml}$ ) were added to the cells and then supplemented with $1.8 \mathrm{ml}$ DMEM. Triplicate wells for the same condition were prepared. Lipofectamine 2000 and FAM-siRNA was used as a positive control, and FAM-siRNA alone was used as a negative control. After $6 \mathrm{~h}$, the transfer efficiency was estimated using a fluorescent microscope.

Gelatin zymography. The gelatin zymography procedure was described in detail in a previous study (12). Briefly, electrophoresis plates for $8 \%$ SDS-PAGE, including $1 \mathrm{~g} / 1$ gelatin, were prepared (Sigma-Aldrich). The samples from the cultured supernatants were prepared using dialysis bags, with a molecular weight cut-off of $35 \mathrm{kDa}$, and incubated in polyethyleneglycol (Sigma-Aldrich) for $40 \mathrm{~min}$ at RT to concentrate the samples. Subsequently, $15 \mathrm{~g}$ concentrated supernatant from each sample was loaded for electrophoresis onto the prepared gel, which was run at $100 \mathrm{~V}$. Subsequently, the gel was rinsed twice with Zymogram A buffer for $30 \mathrm{~min}$ at RT and incubated with Zymogram B buffer overnight. Finally, the gel was stained with Coomassie Brilliant Blue R-250 (0.2\% Coomassie Brilliant Blue R-250, 20\% methanol and 10\% acetic acid) for $2 \mathrm{~h}$ at $37^{\circ} \mathrm{C}$ and destained in methanol to obtain clear bands. The gray values of the bands were analyzed using a Smart Biological Electrophoretic Image Analyzer. The MMP-2 activity was presented as a percentage of the activity of the $12 \mathrm{~h}$ control group.

DAPI staining. The cells were fixed in cold acetone for $20 \mathrm{~min}$, followed by staining with DAPI staining buffer for $10 \mathrm{~min}$ at RT. Following washing with PBS, the cellular morphology was analyzed and images were captured using a fluorescent microscope.

Immunocytochemistry staining. The cells were fixed in cold acetone, and nonspecific antibody binding was blocked with goat serum. This was followed by incubation with the primary antibody, $\alpha$-SMA, or type I collagen monoclonal antibody (1:100) and a secondary antibody, conjugated to biotin and immunoperoxidase with streptavidin at $4^{\circ} \mathrm{C}$. Subsequently, the cells were visualized using DAB and $\mathrm{H}_{2} \mathrm{O}_{2}$, followed by counter-staining with hematoxylin (Sigma-Aldrich), and the intensity of staining was observed under a fluorescent microscope. The negative control was treated with PBS buffer instead of a monoclonal antibody. A total of five images were captured in the left, middle, right, upper and lower fields of each slide. The protein expression levels of $\alpha$-SMA or type I collagen was calculated as the average percentage of positive cells (number of positive cells/number of total cells x 100\%).

Statistical analysis. All experiments were performed at least three times, and the numerical data are presented as the mean \pm standard deviation. Statistical analysis was performed using SPSS 13.0 software. The difference between two groups were evaluated using Student's t-test for independent samples. $\mathrm{P}<0.05$ was considered to indicate a statistically significant difference.

\section{Results}

Physicochemical properties of liposomes. The present study used infrared spectra to examine whether cephalin was bound by VitA. The spectra of cephalin and VitA-cephalin were the same, with the exception of the vibration benzene peak at $1,456.9 \mathrm{~cm}^{-1}$ and an $\mathrm{NH}$-stretching vibration characteristic peak of an amide bond at 3,507.2 $\mathrm{cm}^{-1}$ for VitA-cephalin, which confirmed that VitA bound cephalin via an amide bond (Fig. 1A)

Subsequently, the morphology and stability of the liposomes were examined. The results revealed that unmodified cationic liposomes appeared as white emulsions with light blue opalescence. They dispersed well, and the majority were single chamber and spherical-like, with particles sizes distributed between $100 \mathrm{~nm}$ and $200 \mathrm{~nm}$ (Fig. 1B). No flocculation or sediment were observed on being maintained at RT for 2 months, however, their diameter increased without prominent fusion. Following modification by VitA, the particle size of the cationic liposomes increased, and they exhibited mild aggregation (Fig. 1B). These findings were further confirmed by the results from the Zeta Sizer 3000 laser particle size analyzer. The data indicated that the average size of the cationic liposomes was $148.2 \pm 0.3 \mathrm{~nm}$ and the surface charge, based on the zeta potential measurement, was $+41.67 \mathrm{mV}$, with these values changing to $227.3 \pm 4.1 \mathrm{~nm}$ and $+44.67 \mathrm{mV}$, respectively following modification (Table I). The PDI values revealed an almost monodisperse particle size (Table I). These findings demonstrated that the VitA-lips exhibited a positive charges with good stability.

Identification of MMP-2 siRNA interference efficiency. To obtain higher gene silence efficiency, three interference sequences and corresponding random sequences of MMP-2 were designed. At $36 \mathrm{~h}$ post-transfection of the cells with the VitA-lip-MMP-2 siRNA complexes, the cells were used to evaluate the mRNA expression of MMP-2 using RT-qPCR. The data demonstrated that the interference efficiencies of MMP-2 siRNA-A, siRNA-B and siRNA-C were 58.76, 52.87 and $61.84 \%$, respectively, and no interference effects were observed in the cells treated with VitA-lip only (Fig. 2). These results indicated that the mRNA expression 
Table I. Characterization of liposomes.

\begin{tabular}{|c|c|c|c|c|}
\hline \multirow[b]{2}{*}{ Method } & \multirow{2}{*}{$\frac{\text { TEM }}{\text { Size distribution }(\mathrm{nm})}$} & \multicolumn{3}{|c|}{ Zeta sizer 3000 laser particles analyzer } \\
\hline & & $\mathrm{Z}$-average diameter $(\mathrm{nm})$ & Polydispersity & Zeta potential $(\mathrm{mV})$ \\
\hline Liposome & $114.34 \pm 36.94$ & $148.2 \pm 0.3$ & $0.162 \pm 0.014$ & +41.67 \\
\hline VitA-lips & $155.77 \pm 39.00$ & $227.3 \pm 4.1$ & $0.171 \pm 0.025$ & +44.67 \\
\hline
\end{tabular}

Data are expressed as the mean \pm standard deviation. VitA, vitamin A; lips, liposomes; TEM, transmission electron microscopy.

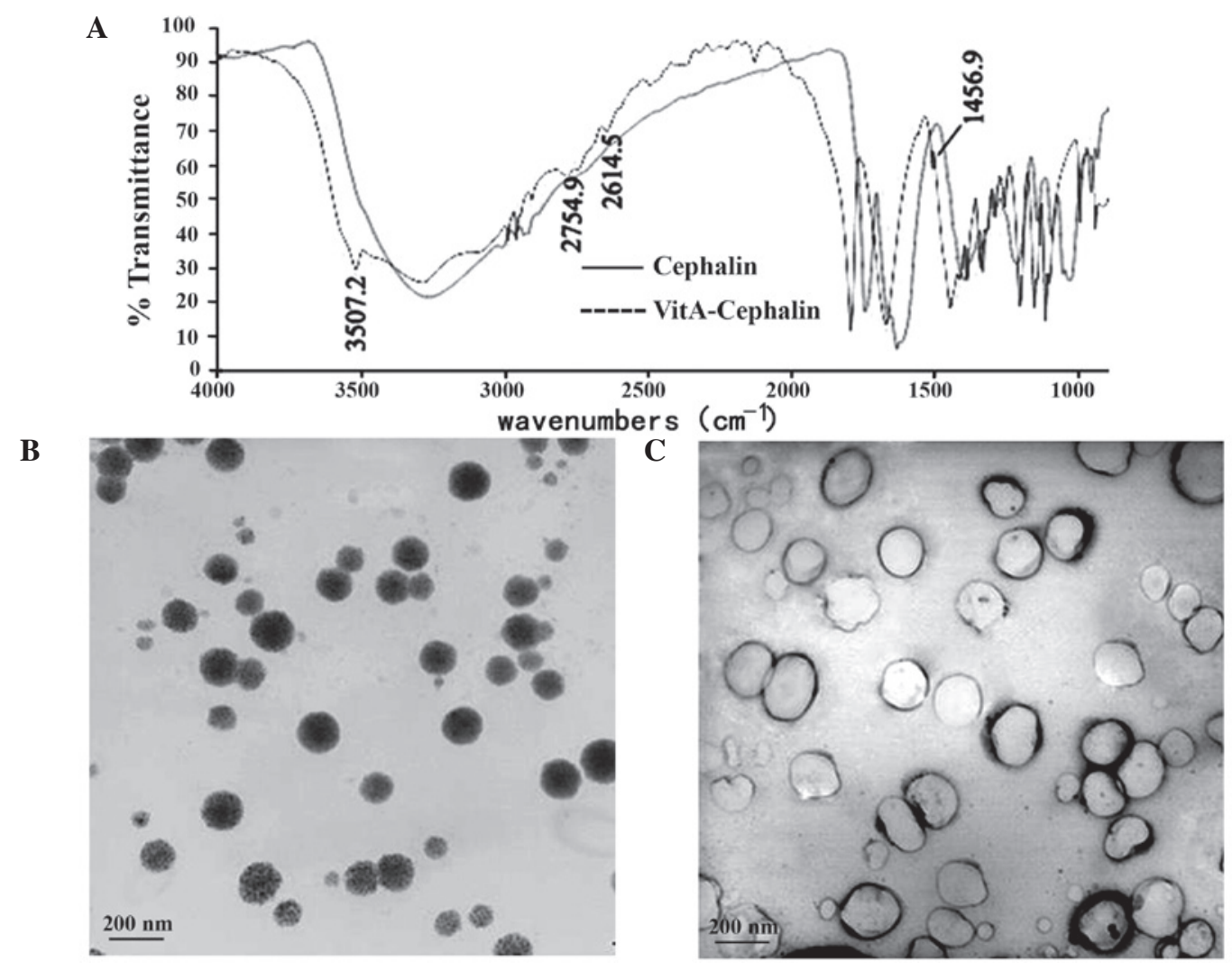

Figure 1. Characterization of cationic liposomes. (A) Stretching vibration peaks of cephalin modified with VitA on the Fourier infrared spectrum. Transmission electron microscopic images of (B) cationic liposomes and (C) VitA-Lips. VitA, vitamin A.

levels of the target gene in the three interference groups were significantly different compared with that in the untreated control or VitA-lip group. Among these, MMP-2 siRNA-C exhibited the highest silencing efficiency and was used for the subsequent experiments.

siRNA-binding capacity of theliposomes. To determine the siRNA-binding capability of the liposomes, agarose gel electrophoresis was performed. This process demonstrated that the cationic liposomes were bound to more siRNA when the ratio of cationic liposome to siRNA was increased. The cationic liposomes completely encapsulated MMP-2 siRNA when the ratio of cationic liposomes to siRNA was $\geq 1$. Following modifiication by VitA, the cationic liposomes effectively integrated with siRNA when the ratio was $\geq 5$. Therefore, the cationic liposomes exhibited stable binding capability, which was efficient following modification by VitA (Fig. 3).
Transfection efficiency of VitA-lip-MMP-2 siRNA. Prior to examining the transfection efficiency of the VitA-lip-MMP-2 siRNA complexes, their cellular toxicity was examined using an MTT assay. Following treatment of the cells with pure liposomes or with the complexes containing the various mass ratios of liposome to siRNA $(5: 1,10: 1$ and 15:1), no cytotoxicity was observed in the HSC-T6 cells whether the complexes contained the liposomes or VitA-lips (Fig. 4E). As expected, the cytotoxicity was liposome concentration-dependent (Fig. 4E). Although decreased cell viability was observed in the HSC-T6 cells transfected with the complexes, they remained at $\sim 80 \%$, despite a liposome/siRNA ratio of 15:1 (Fig. 4E).

Subsequently, the cells were transfected with cationic liposome/MMP-2 siRNA complexes with different mass ratios and, after $6 \mathrm{~h}$, green fluorescence was observed inside the cells exposed to the complexes, however no fluorescence was observed when cells were treated with MMP-2 siRNA alone (Fig. 4A-D). Among the cell groups, the highest transfection 
A

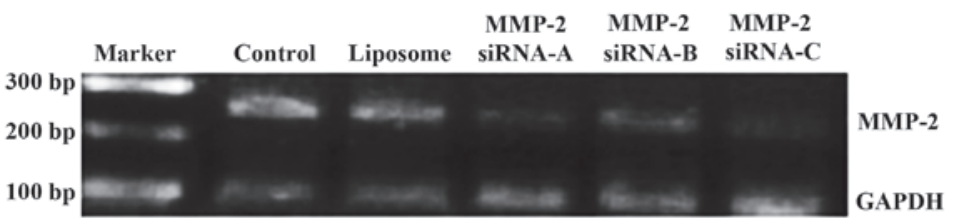

B

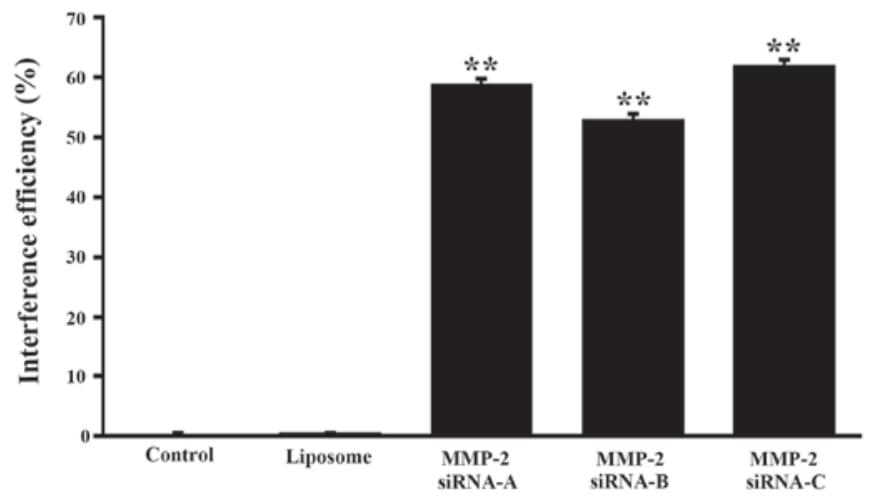

Figure 2. Interference efficiency of VitA-lip-MMP-2 siRNA complexes. The mRNA expression of MMP-2 was examined using reveres transcription-quantitative polymerase chain reaction in HSC-T6 cells. The cells were exposed to normal complete medium (control), liposomes and VitA-lip-MMP-2 siRNA complexes containing three designed MMP-2 siRNA-A/B/C sequences. (A) Images of gelatin zymography. (B) Quantitative analysis of densitometry indicating MMP-2 activity. The data indicated that the VitA-lip-MMP-2 siRNA complexes containing the MMP-2 siRNA-C sequence exhibited the highest interference efficiency. All the experiments were repeated at least three times. Representative images from one of the experiments are shown. Data are expressed as the mean \pm standard deviation. ${ }^{* *} \mathrm{P}<0.01$, compared with the untreated control. MMP-2, matrix metalloproteinase 2; siRNA, small interference RNA; VitA, vitamin A; lip, liposome.

A

$20 \mathrm{bp}$

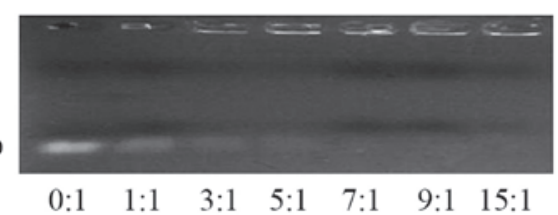

B

$20 \mathrm{bp}$

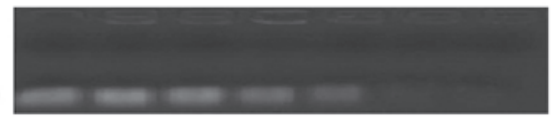

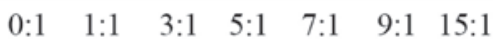

Mass ratios Liposome/SiRNA

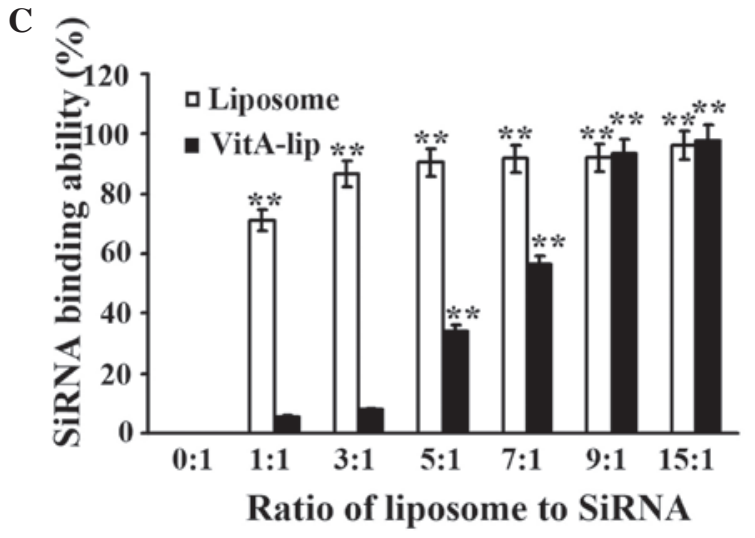

Figure 3. siRNA-binding capacity of cationic liposomes. Different mass ratios of cationic liposomes or VitA-Lips were mixed with siRNA, and a gel retardation assay was performed. (A) cationic liposomes; (B) VitA-Lips; (C) Quantitative analysis of densitometry in A and B. The mass ratios of liposome/siRNA were 0:1, 1:1, 3:1, 5:1, 7:1, 9:1 and 15:1 in lanes 1-7, respectively. The data are representative of three replicate experiments. Data are expressed as the mean \pm standard deviation. ${ }^{* *} \mathrm{P}<0.01$, compared with the control (mass ratio, 0:1). siRNA, small interference RNA; VitA, vitamin A. efficiency was detected when the ratio of liposome/siRNA was 5:1 prior to modification. Following modification with VitA, the most efficient transfection was achieved at a liposome/siRNA ratio of 7:1 (data not shown). These findings indicated that the transfection efficiency increased markedly following modification. However, the cellular toxicity was dependent on the mass ratio of liposome/siRNA, of which a ratio of 7:1 was selected for the subsequent experiments.

Silencing efficiency of MMP-2 induced by VitA-lip-MMP-2 siRNA. To further optimize the MMP-2 interference, the HSCs cells were transfected with VitA-lip-MMP-2 siRNA for 12, 24, 36, 48 and $72 \mathrm{~h}$. The activities of MMP-2 in the supernatants were then detected using zymography. As shown in Fig. 4, the gene interference gradually increased between 12 and $48 \mathrm{~h}$, peaked at $48 \mathrm{~h}$ and weakened at $72 \mathrm{~h}$ (Fig. 5).

The effect of VitA-lip-MMP-2 siRNA on the cellular behavior of HSCs. To investigate the effect of VitA-lip-MMP-2 siRNA complexes on the cellular behavior of HSC-T6 cells, DAPI staining was performed to examine whether apoptosis was induced. Following transfection of the HSCs with VitA-lip-MMP-2 siRNA complexes for $48 \mathrm{~h}$, the cells were stained with DAPI staining solution. No differences in apoptosis were observed in the cells in the interference groups, compared with those of the control groups (Fig. 6), which suggested that the decreased viability of the HSC-T6 cells was due to the downregulated cell activation, and not apoptosis.

To detect the activation of the HSC-T6 cells, the present study performed immunostaining with specific antibodies to $\alpha$-SMA and type I collagen, with positive cells presenting 

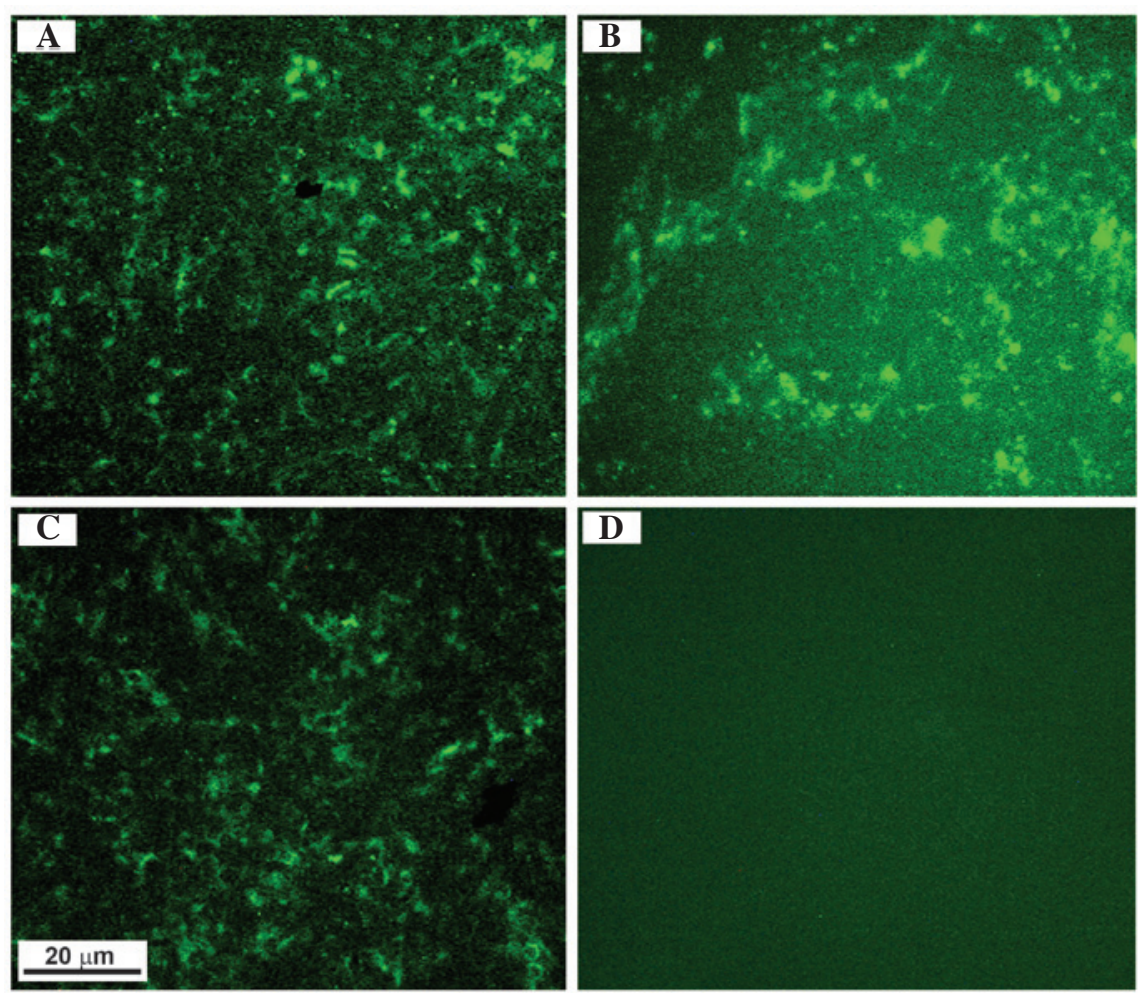

$\mathbf{E}$

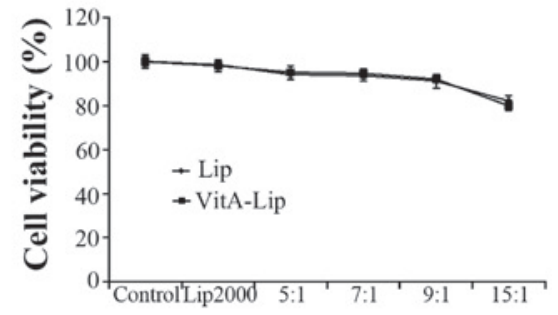

Figure 4. Transfection efficiency and cytotoxicity of VitA-lip-MMP-2 siRNA complexes. Cells were plated on six-well plates overnight and treated with (A) Lipofectamine 2000, (B) siRNA capsuled with cationic liposomes, (C) FAM-siRNA capsuled with VitA-lip and (D) FAM-MMP-2 siRNA After 6 h, the transfection efficiency was observed using a fluorescence microscope. (E) Cell viability was detected using an MTT assay following transfection of the cells with liposomes, or with MMP-2 siRNA encapsulated with unmodified cationic liposomes or VitA-lips. All experiments were repeated at least three times. Representative images from one of the experiments are shown. Data are expressed as the mean \pm standard deviation. MMP-2, matrix metalloproteinase 2; siRNA, small interference RNA; VitA, vitamin A.
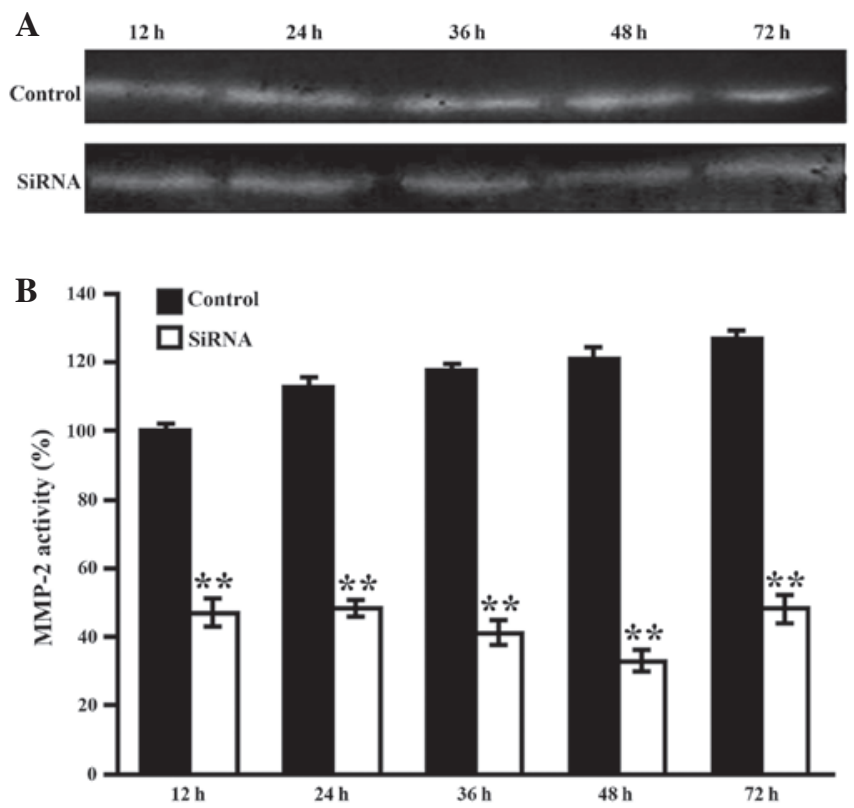

Figure 5. Interference efficiency of the VitA-lip-MMP-2 siRNA complexes The MMP-2 activity of the cell culture supernatants was evaluated using zymography following transfection of the cells with VitA-lip-MMP-2 siRNA complexes for 12, 24, 36, 48 and $72 \mathrm{~h}$. (A) Images of gelatin zymography. (B) Quantitative analysis of densitometry indicating MMP-2 activity MMP-2 activity is presented as a percentage of the activity of the $12 \mathrm{~h}$ control group. All the experiments were repeated at least three times. Representative images from one of the experiments are shown. Data are expressed as the mean \pm standard deviation. ${ }^{* *} \mathrm{P}<0.01$, compared with the corresponding control. MMP-2, matrix metalloproteinase 2; siRNA, small interference RNA; VitA, vitamin A. diffusely distributed brown-yellow granules in the cytoplasm. The results demonstrated that the expression of $\alpha$-SMA was significantly decreased and the number of positive cells was reduced following treatment with MMP-2 siRNA, compared with that of the control groups (Fig. 7). The same expression pattern was observed for type I collagen (Fig. 7).

\section{Discussion}

Hepatic fibrosis is the final stage of all chronic hepatic diseases and may develop into cirrhosis and hepatic carcinoma, and finally induce liver failure (13). The sustained secretion of ECM is a prerequisite of hepatic fibrogenesis, and HSCs are vital cells, which produce ECM during the development of hepatic fibrosis $(13,14)$. The activation of HSCs has been considered an important step for the formation and development of fibrosis $(3,9,11,15)$. In the healthy liver, HSCs are in a quiescent state and are involved in the metabolism of VitA. Under physiological conditions, HSCs do not express $\alpha$-SMA, and exhibit low proliferative ability and collagen secretion $(10,11,16)$. However, when exposed to injury, HSCs are activated and transformed into a fibroblast phenotype $(10,11)$. HSCs lose VitA in the cytoplasm, but express cytokines, receptors, $\alpha$-SMA and ECM, including type I collagen, and proliferate rapidly $(11,16,17)$. HSCs are not only key cells in ECM secretion, they also crucial cells in the generation of MMP (18). Previous reports suggest that hepatic fibrosis is reversible $(1-3,6,14)$. However, no effective drugs have been 


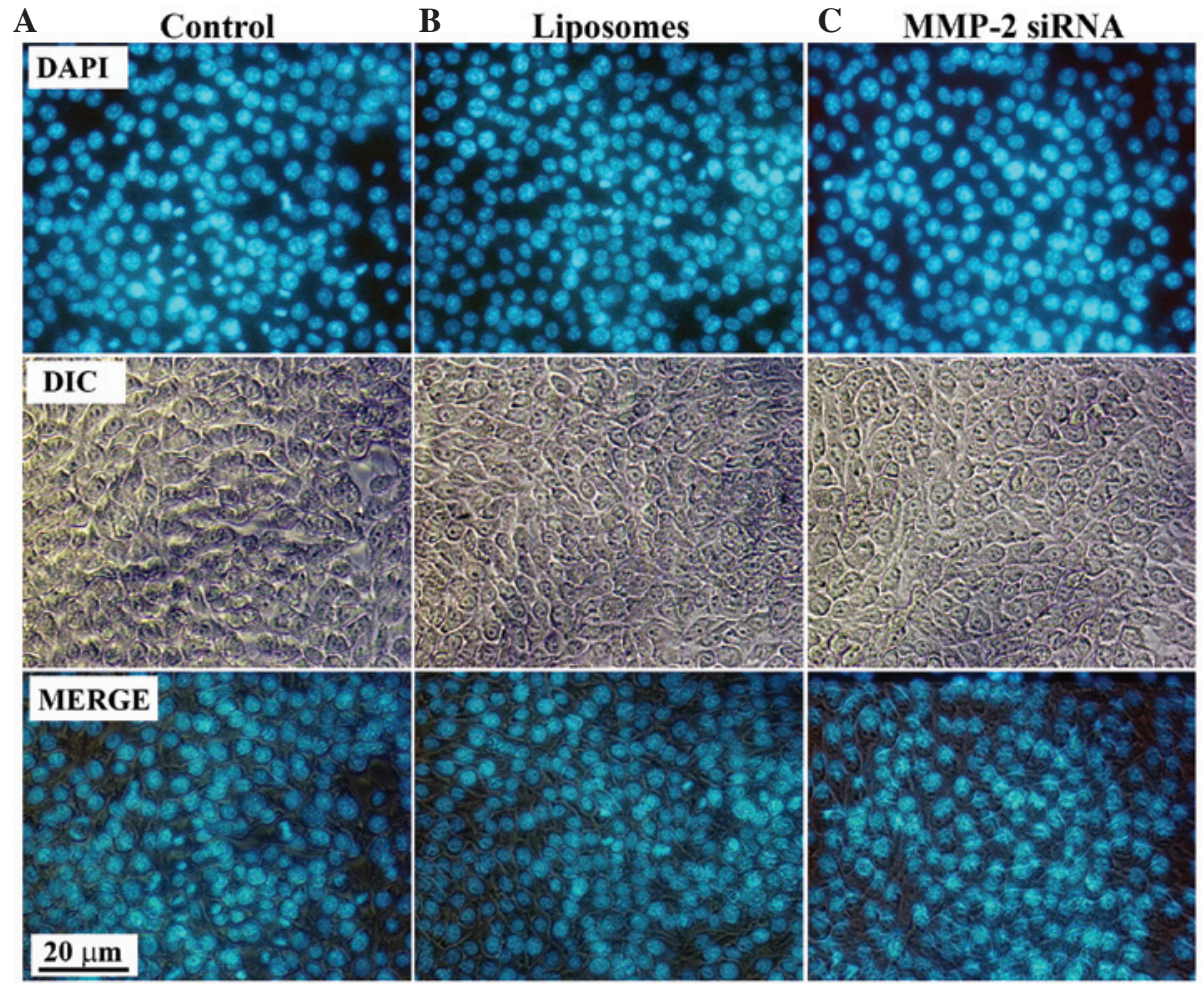

Figure 6. Effect of VitA-lip-MMP-2 siRNA complexes on apoptosis in the HSC-T6 cells. HSC-T6 cells stained using 4',6-diamidino-2-phenylindole solution exhibited no clear apoptosis following transfection with MMP-2 siRNA for 48 h. (A) Standard culture medium; (B) liposomes; (C) VitA-lip MMP-2 siRNA. MMP-2, matrix metalloproteinase 2; siRNA, small interference RNA; VitA, vitamin A; DIC, differential interference contrast.

A

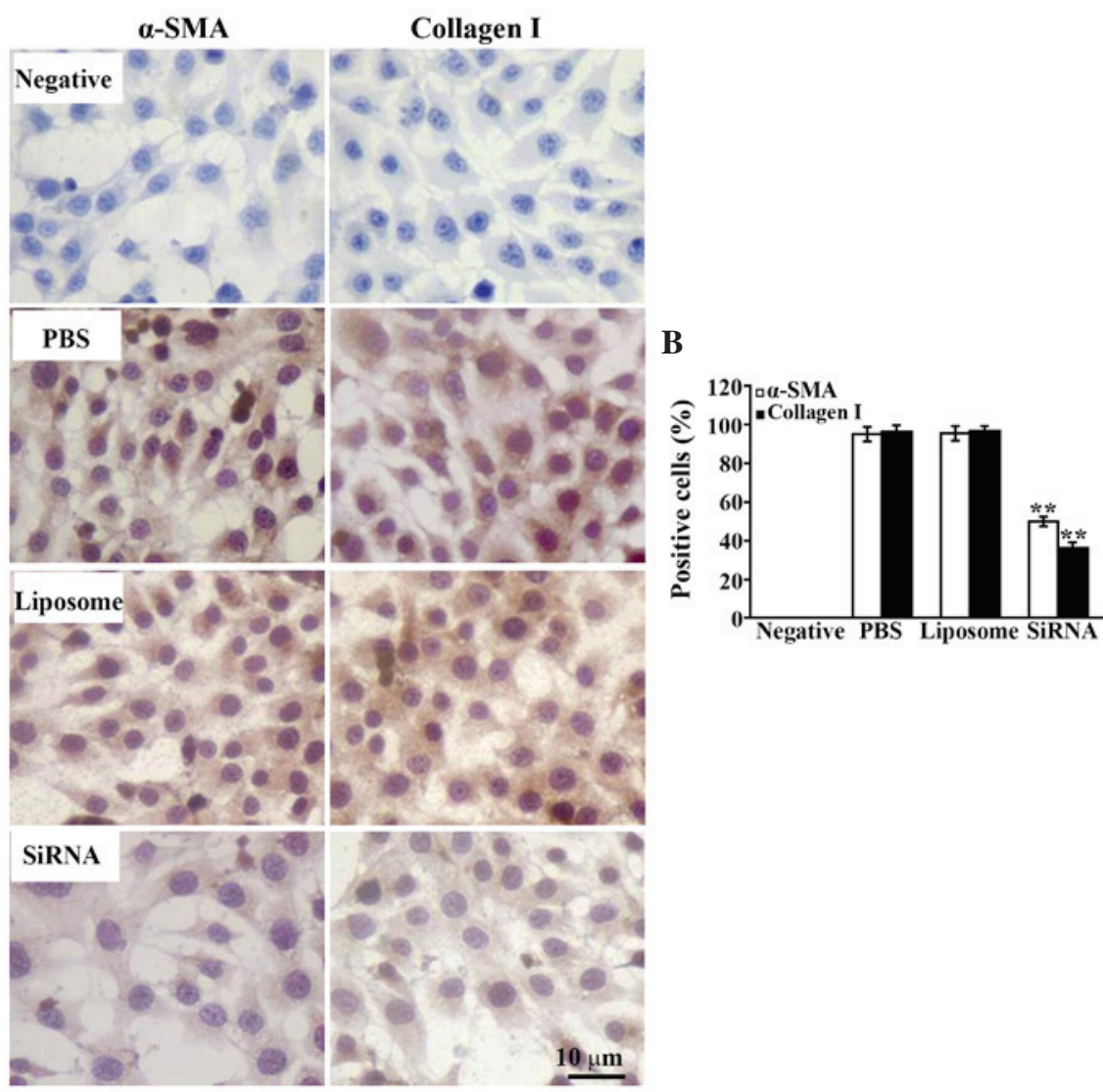

Figure 7. Effect of VitA-lip-MMP-2 siRNA complexes on HSC-T6 cell activation. The markers of activated HSCs, $\alpha$-SMA and type I collagen, were evaluated using immunocytochemical staining in HSC-T6 cells treated with liposomes containing MMP-2 siRNA. (A) Representative fluorescent images. (B) Quantitative analysis of the changes in fluorescence intensity, indicating the degree of the activation in the HSC-T6 cells. Data are expressed as the mean \pm standard deviation. ${ }^{* *} \mathrm{P}<0.01$, compared with the untreated (negative group) or liposome group. HSCs, hepatic stellate cells; PBS, phosphate-buffered saline; VitA, vitamin A; siRNA, small interference RNA; SMA, smooth muscle actin. 


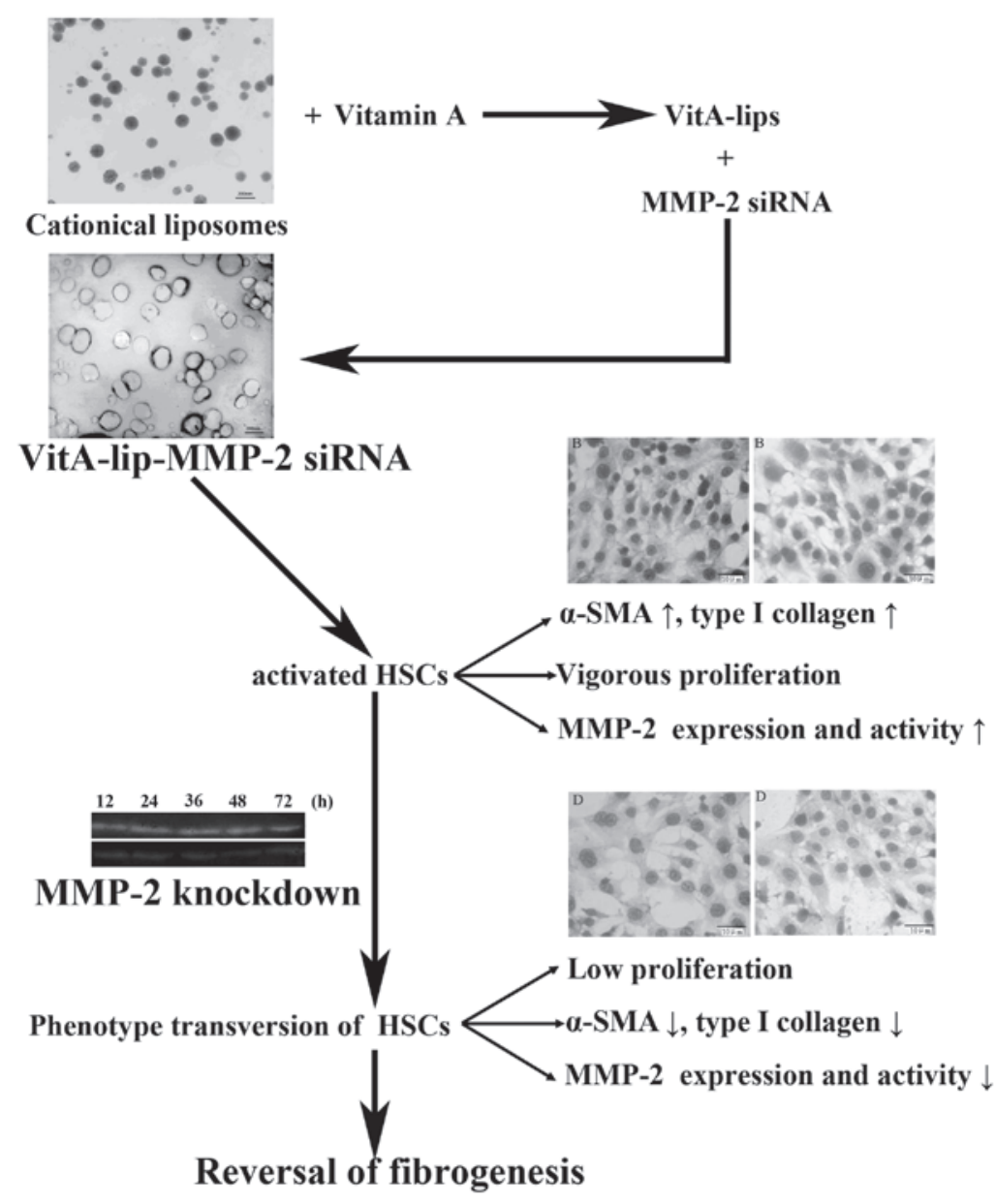

Figure 8. Schematic illustration of the association among HSCs, MMP-2 and fibrogenesis. At the fibrogenesis stage, hepatic stellate cells proliferate rapidly, secrete abundant MMP-2 and express markers of activated HSCs ( $\alpha$-SMA and type I collagen). However, when MMP- 2 was downregulated by the constructed liposomes carrying MMP-2 siRNA, the viability of the HSCs decreased and expression levels of $\alpha$-SMA and type I collagen were reduced. These results indicated that silencing MMP-2 reversed fibrogenesis. HSCs, hepatic stellate cells; PBS, phosphate-buffered saline; VitA, vitamin A; siRNA, small interference RNA; SMA, smooth muscle actin.

developed against the formation of fibrosis in chronic liver disease due to the shortage of specific targets and drugs, and inevitable side effects.

MMP-2 is an important collagenase of the matrix metalloproteinase family and is produced by several types of cell, including activated HSCs (19). At the early stage of fibrosis, HSCs secrete substantial MMP-2 and degrade abundant type IV collagen around the HSCs, which promotes the further activation and proliferation of HSCs $(11,12,16,20,21)$. The activated HSCs secrete more collagen and MMP-2, following which MMP-2 conversely accelerates HSC activation, which constitutes a malignant feedback loop. MMP-2 also contributes to angiogenesis, vascular remodeling and hepatic sinusoid capillarization, which aggravates the progression of fibrosis (20). However, in the naturally decaying stage, MMP-2 activity is increased (22). This indicates that MMP-2 promotes the development of fibrosis in the early stage, but induces the elimination of fibrosis in the late stage. These findings can assist in developing specific drugs for fibrosis by regulating the expression and activity of MMP-2 during different periods of hepatic fibrosis.

RNAi is a gene silencing tool of substantial functionality. To silence a specific gene, specific homogenous mRNA is degraded using double-stranded RNA. This technique has the advantage of high efficiency and specificity and is widely used for investigating prophylaxis and treatment of diseases (23). Considering the vital functions of activated HSCs in the development of hepatic fibrosis, several studies have examined the regulation of gene expression by siRNA interference, which further inhibits activation and proliferation, promotes apoptosis and increases degradation of the ECM in HSCs. The findings of these studies suggest that HSCs are promising targets in identifying effective drugs against hepatic fibrosis. However, the shortage of specificity in RNAi in vivo has limited its application. Thus, based on the specific receptor and regulating sequences on the cell surface, a number of vectors targeting HSCs have been identified, including mannose-targeted liposomes and folic acid-targeted liposomes (24). These vectors specifically deliver siRNA to HSCs, which protects healthy hepatocytes. Furthermore, the higher efficiency of siRNA transfer results in more effective therapy. Large numbers of VitA receptors are expressed on the cellular membrane of HSCs (11), and Sato et al confirmed its specificity and effectiveness in vivo (25). Therefore, the present study constructed VitA-coupled cationic liposomes to deliver MMP-2 siRNA, and the data confirmed that the vector 
efficiently conveyed siRNA into the HSCs and inhibited the gene expression of MMP-2. However, the specificity of the vector requires further investigation in vivo.

The HSC-T6 cell line, an activated HSC model, has been used as a target cell in several studies on hepatic fibrosis. HSC-T6 cells present the features of hepatic stellate cells and activation phenotypes, including vigorous proliferation, abundant expression levels of $\alpha$-SMA and type I collagen, and fibroblast-like morphology $(3,26)$. A study by Kawada (27) found that MMP-2 promotes the activation and proliferation of HSCs, activates the expression of $\alpha$-SMA, a marker of HSC activation, and produces increased ECM, predominantly type I collagen. In the present study, HSCs activation and the expression of type I collagen were reduced following treatment with MMP-2 siRNA. The results demonstrated that the MMP-2 siRNA-transfected HSCs were smaller, and the ratio of nucleus to plasma was reduced. In addition, the cell viability was downregulated, which suggested that decreased activity and expression of MMP-2 reduced the the proliferation rate of the HSCs, however, these findings were not associated with apoptosis. Therefore, the present study hypothesized that the reduction in the viability of the HSCs may have been caused by MMP-2 siRNA-induced phenotype transversion of the activated HSCs. The immunostaining confirmed this hypothesis, as the protein expression levels of $\alpha$-SMA and type I collagen were significantly decreased in MMP-2 siRNA-treated HSCs. However, whether the weakened viability of the HSCs is associated with the induction of senescence requires further investigation.

In conclusion, the present study successfully constructed a VitA-coupled cationic liposome vector, which effectively delivered MMP-2 siRNA to the HSCs. Following transfection with the vector, the expression and activity of MMP-2 in the HSCs were prominently downregulated. Based on these changes, the activation and proliferation of HSCs were decreased, and the secretion of type I collagen in the HSCs cells was significantly reduced (Fig. 8). These findings present a novel direction in the targeted prevention of hepatic fibrosis. However, further investigations are required to elucidate the application of this system in vivo.

\section{Acknowledgements}

This study was supported by grants from the National Basic Research Program, People's Republic of China (973 Program; grant. no. 2011CB933404), the National Natural Science Foundation, People's Republic of China (grant. no. 30470780), and the Specialized Research Fund for the Doctoral Program of Higher Education (grant. no. 20090092110053).

\section{References}

1. Povero D, Busletta C, Novo E, et al: Liver fibrosis: a dynamic and potentially reversible process. Histol Histopathol 25: 1075-1091, 2010.

2. Ismail MH and Pinzani M: Reversal of hepatic fibrosis: pathophysiological basis of antifibrotic therapies. Hepat Med 3: 69-80, 2011.

3. Novo E, Cannito S, Paternostro C, Bocca C, Miglietta A and Parola M: Cellular and molecular mechanisms in liver fibrogenesis. Arch Biochem Biophys 548: 20-37, 2014.
4. Hu YB, Li DG and Lu HM: Modified synthetic siRNA targeting tissue inhibitor of metalloproteinase-2 inhibits hepatic fibrogenesis in rats. J Gene Med 9: 217-229, 2007.

5. Mormone E, George J and Nieto N: Molecular pathogenesis of hepatic fibrosis and current therapeutic approaches. Chem Biol Interact 193: 225-231, 2011.

6. Radbill BD, Gupta R, Ramirez MC, et al: Loss of matrix metalloproteinase-2 amplifies murine toxin-induced liver fibrosis by upregulating collagen I expression. Dig Dis Sci 56: 406-416, 2011.

7. Kong D, Zhang F, Zhang Z, Lu Y and Zheng S: Clearance of activated stellate cells for hepatic fibrosis regression: molecular basis and translational potential. Biomed Pharmacother 67: 246-250, 2013.

8. Balazs DA and Godbey W: Liposomes for use in gene delivery. J Drug Deliv 2011: 326497-326508, 2011.

9. Kocabayoglu P and Friedman SL: Cellular basis of hepatic fibrosis and its role in inflammation and cancer. Front Biosci (Schol Ed) 5: 217-230, 2013

10. Puche JE, Saiman Y and Friedman SL: Hepatic stellate cells and liver fibrosis. Compr Physiol 3: 1473-1492, 2013.

11. Senoo H, Yoshikawa K, Morii M, Miura M, Imai K and Mezaki Y: Hepatic stellate cell (vitamin A-storing cell) and its relative-past, present and future. Cell Biol Int 34: 1247-1272, 2010.

12. Fan RH, Chen PS, Zhao D and Zhang WD: Hypoxia induced by $\mathrm{CoCl} 2$ influencing the expression and the activity of matrix metalloproteinase-2 in rat hepatic stellate cells. Zhonghua Gan Zang Bing Za Zhi 15: 654-657, 2007 (In Chinese).

13. Lee UE and Friedman SL: Mechanisms of hepatic fibrogenesis. Best Pract Res Clin Gastroenterol 25: 195-206, 2011.

14. Kumar M and Sarin SK: Is cirrhosis of the liver reversible? Indian J Pediatr 74: 393-399, 2007.

15. Gressner AM: Transdifferentiation of hepatic stellate cells (Ito cells) to myofibroblasts: a key event in hepatic fibrogenesis. Kidney Int Suppl 54 (Suppl): 39-45, 1996.

16. Senoo H, Hata R, Nagai Y and Wake K: Stellate cells (vitamin A-storing cells) are the primary site of collagen synthesis in non-parenchymal cells in the liver. Biomed Res 5: 451-458, 1984.

17. Enzan H, Himeno H, Iwamura S, et al: Immunohistochemical identification of Ito cells and their myofibroblastic transformation in adult human liver. Virchows Arch 424: 249-256, 1994.

18. Schuppan D and Afdhal NH: Liver cirrhosis. Lancet 371: 838-851, 2008

19. Arthur MJ, Friedman SL, Roll FJ and Bissell DM: Lipocytes from normal rat liver release a neutral metalloproteinase that degrades basement membrane (type iv) collagen. J Clin Invest 84: 1076-1085, 1989

20. Li J, Niu JZ, Wang JF, Li Y and Tao XH: Pathological mechanisms of alcohol-induced hepatic portal hypertension in early stage fibrosis rat model. World J Gastroenterol 11: 6483-6488, 2005.

21. Li J, Fan R, Zhao S, et al: Reactive oxygen species released from hypoxic hepatocytes regulates MMP-2 expression in hepatic stellate cells. Int J Mol Sci 12: 2434-2447, 2011.

22. Domitrović R, Jakovac H, Marchesi VV and Blažeković B: Resolution of liver fibrosis by isoquinoline alkaloid berberine in CCl4-intoxicated mice is mediated by suppression of oxidative stress and upregulation of MMP-2 expression. J Med Food 16: 518-528, 2013

23. Schroeder A, Levins CG, Cortez C, Langer R and Anderson DG: Lipid-based Nano therapeutics for siRNA delivery. J Intern Med 267: 9-21, 2010

24. Wasungu L and Hoekstra D: Cationic lipids, lipoplexes and intracellular delivery of genes. J Control Release 116: 255-264, 2006.

25. Sato Y, Murase K, Kato J, et al: Resolution of liver cirrhosis using vitamin A-coupled liposomes to deliver siRNA against a collagen specific chaperone. Nat Biotechnol 26: 431-442, 2008.

26. Vogel S, Piantedosi R, Frank J, et al: An immortalized rat liver stellate cell line (HSC-T6): a new cell model for the study of retinoid metabolism in vitro. J Lipid Res 41: 882-893, 2000.

27. Kawada N: Evolution of hepatic fibrosis research. Hepatol Res 41: 199-208, 2011. 